Résumés des conférences et travaux

\title{
Relations internationales et diplomatie (1300-1700)
}

\section{Stéphane Péquignot}

\section{OpenEdition}

\section{Journals}

\section{Édition électronique}

URL : https://journals.openedition.org/ashp/4448

DOI : $10.4000 /$ ashp.4448

ISSN : 1969-6310

\section{Éditeur}

Publications de l'École Pratique des Hautes Études

\section{Édition imprimée}

Date de publication : 1 septembre 2021

Pagination : 281-287

ISSN : 0766-0677

\section{Référence électronique}

Stéphane Péquignot, «Relations internationales et diplomatie (1300-1700) », Annuaire de l'École ; DOI : https://doi.org/10.4000/ashp.4448 


\title{
RELATIONS INTERNATIONALES ET DIPLOMATIE $(1300-1700)$
}

\author{
Directeur d'études : M. Stéphane PÉQUiGnot
}

Programme de l'année 2018-2019: I. Les lettres de créance en Occident (à partir du XIII siècle). - II. L'expérience de l'incertitude dans les échanges diplomatiques : sources normatives, chroniques (France, péninsule Ibérique, Empire, Italie).

\section{Les lettres de créance en Occident (à partir du XIII siècle)}

Ce nouveau séminaire a été ouvert par une mise en situation historiographique générale. Après une longue période de désaveu, en France tout particulièrement, l'histoire de la diplomatie et des relations internationales suscite depuis bientôt une trentaine d'années un très vif regain d'intérêt. Les premières séances ont permis de prendre la mesure des principales voies empruntées par la recherche récente, de mettre en évidence les contours d'un domaine dont la définition fait encore débat, et d'expliquer l'adoption d'une périodisation large, mais non systématique, du XIII ${ }^{\mathrm{e}}$ au $\mathrm{XVII}^{\mathrm{e}}$ siècle. Les sources disponibles sont alors bien plus considérables et diversifiées que pour la période précédente. Il en résulte un observatoire remarquable pour l'étude des conceptions et des pratiques de la diplomatie, des négociations et des relations internationales.

Le premier thème retenu cette année a été celui des lettres de créance, des pièces dont l'usage joue un rôle essentiel dans les échanges diplomatiques à partir $\mathrm{du} \mathrm{XIII}^{\mathrm{e}}$ siècle. Après un bilan général des travaux effectués à leur sujet, depuis les analyses fondatrices de René de Maulde-la-Clavière sur la diplomatie de Louis XII jusqu'aux travaux plus récents de Donald E. Queller et de Pierre Chaplais, puis de Francesco Senatore et Isabella Lazzarini sur l'Italie, l'analyse a d'abord porté sur le vocabulaire ambivalent de la «créance» ou «credence», qui pouvait renvoyer tout autant à l'accréditation qu'à l'instruction, avant d'envisager la composition, la langue, la matérialité et la destinée archivistique de ces lettres.

Dans un second temps, l'accent a été mis sur les audiences lors desquelles les lettres de créance sont présentées, sur les gestes effectués et les discours proférés à cette occasion. En prenant pour cas d'étude les diplomaties française, castillane et aragonaise du $\mathrm{XIII}^{\mathrm{e}}$ siècle au début du XVI ${ }^{\mathrm{e}}$ siècle, l'enquête a été menée dans trois directions complémentaires. Il s'est agi tout d'abord de rechercher la place réservée aux lettres de créance dans les documents à caractère normatif et prescriptif. Les miroirs des princes, les premiers traités sur les ambassadeurs, notamment celui de Bernard de Rosier, les ordonnances de cour et les formulaires de chancellerie, pourtant nombreux de part et d'autre des Pyrénées, n'ont livré que fort peu d'éléments au sujet des lettres de créance. Les instructions remises aux ambassadeurs se sont avérées plus parlantes. L'analyse a porté sur les plus anciennes instructions françaises, sur un corpus inédit de plusieurs centaines d'instructions bâillées par les rois d'Aragon du XIII ${ }^{\mathrm{e}}$ siècle $\mathrm{au} \mathrm{XV}^{\mathrm{e}}$ siècle, ainsi que sur de nombreuses instructions des Rois Catholiques à leurs 
ambassadeurs. Les lettres de créance y occupent une place secondaire, qui devient toutefois tendanciellement plus importante au $\mathrm{XV}^{\mathrm{e}}$ siècle. Dans l'immense majorité des cas, les instructions, centrées sur les paroles et les discours, ne précisent nullement les gestes accomplis lors de la remise des lettres de créance. Pour ces cérémonies comme de manière plus générale pour l'action des ambassadeurs dans les cours étrangères, les gestes qu'ils doivent accomplir sont le plus souvent confiés à leur seule appréciation. Leur maîtrise forme longtemps un savoir tacite, implicite, que l'on ne juge pas nécessaire de codifier par écrit.

L'analyse a dans un deuxième temps été déplacée vers les sources narratives. Des lettres de créance ont pu être décelées dans plusieurs chroniques sous des formes plus ou moins abrégées. Elles sont parfois mentionnées à l'occasion d'événements à caractère diplomatique. Tel est le cas dans la Chronique du roi Pedro Ir de Fernão Lopes, dans le Livre du roi Pierre IV d'Aragon, dans la Crónica du roi de Castille Ferdinand IV (r. 1295-1312), partie de la Crónica de los tres reyes, ainsi que dans la Crónica du chancelier castillan Pedro López de Ayala, qui mentionne à six reprises des lettres de créance, et dans la Crónica de Juan II quelques décennies plus tard. Dans ces textes, la remise des lettres s'accompagne de gestes et plus généralement d'actes que les documents à caractère prescriptif passent sous silence. La révérence, le baiser de la main, une pratique particulièrement répandue en péninsule Ibérique, ont ainsi pu faire l'objet d'analyses détaillées. En contrepoint, l'examen des œuvres de Froissart et de Philippe de Commynes a confirmé l'importance accordée par les contemporains à la remise des lettres de créance, l'existence de gestes codifiés à accomplir et une double valeur accordée à cet acte, un moment de seuil dont le franchissement permet à l'ambassadeur de devenir reconnu comme tel par le destinataire de sa mission, et, dans le même temps, une mise à l'épreuve de la qualité de la relation entre les protagonistes de l'échange diplomatique. Les gestes et les mots de chacun sont en effet observés et interprétés avec une grande attention, à la recherche de signes fiables pour saisir le cours ultérieur des interactions. Ces caractéristiques ont été confirmées par l'analyse des mentions de lettres de créance dans plusieurs correspondances d'ambassadeurs du XVI ${ }^{\mathrm{e}}$ siècle (Lope Hurtado de Mendoza, Jean du Bellay, Gutierrez Gomez de Fuensalida).

L'enquête a pour finir été étendue aux sources iconographiques, jusqu'à présent trop souvent négligées par les spécialistes de l'histoire de la diplomatie et des relations internationales, en particulier pour les $\mathrm{XIII}^{\mathrm{e}}-\mathrm{XV}^{\mathrm{e}}$ siècles. Trois dossiers ont été examinés avec une attention particulière : la Cronaca figurata de Giovanni Villani, où sont représentées des ambassades échangées à l'occasion des Vêpres siciliennes; plusieurs témoins de Froissart et un échantillon de 31 manuscrits enluminés des Grandes chroniques de France. Leur étude a notamment mis en évidence le rôle essentiel de l'écrit dans les miniatures représentant des audiences d'ambassadeurs alors conçues comme une part inhérente du métier de roi et une occasion, en retenant les ambassadeurs venus de loin ou dépêchés par des princes très puissants, de souligner la renommée et la reconnaissance dont jouissent les rois représentés. De la sorte, tout en faisant apparaître les diverses valeurs performatives associées à la remise des lettres de créance, cette partie du séminaire a permis d'identifier et d'analyser le répertoire complexe des gestes réalisés lors des cérémonies - la révérence, le salut, l'accolade, 
le baiser sur le pied ou sur la main, le fait d'ôter son chapeau, de relever quelqu'un, les mouvements des mains et des regards, etc. -, un répertoire qui se cristallise en usages, différents selon les cours, et donne matière à des connotations et inflexions multiples, à des signes auxquels les participants à ces échanges et ceux qui écrivent à leur sujet se montrent très sensibles.

\section{L'expérience de l'incertitude dans les échanges diplomatiques : sources normatives, chroniques (France, péninsule Ibérique, Empire, Italie)}

Rois, princes, ambassadeurs et autres acteurs des échanges diplomatiques se situent, écrivent, et doivent souvent agir dans un contexte marqué par un horizon d'incertitude. Après un examen attentif de l'historiographie, qui a permis de déceler les traces d'un frémissement autour de cette dimension importante de l'action diplomatique ordinairement passée sous silence ${ }^{1}$, puis une mise au point sur les notions employées, l'enquête a dans un premier temps été orientée vers les écrits sur l'ambassadeur et l'art de négocier, dans le but de réunir des éléments permettant de discerner des évolutions dans la façon d'appréhender l'incertitude et le doute dans les échanges diplomatiques.

Les enjeux de typologie (problématique) des écrits sur l'ambassadeur et l'art de négocier une fois précisés, quatre textes ont été examinés en détail : l'Ambaxiatorum brevilogus de Bernard de Rosier (1436), le De officio legati d'Étienne Dolet (1541), un ouvrage de Konrad Braun, Les cinq livres sur les ambassades à destination de ceux qui sont totalement appliqués à la Chose publique, ou très utiles à ceux qui remplissent une quelconque magistrature, et agréables à la lecture (1548), puis El embajador (1620) de Juan Antonio de Vera.

Pour Bernard de Rosier, dont le but est de rehausser la dignité de l'office d'ambassadeur, ses qualités morales et éthiques, mais aussi de suggérer de façon pragmatique les moyens de parvenir à une telle fin, l'incertitude et l'opposition entre le certain et l'incertain se sont avérées jouer un rôle non négligeable. Il existe selon cet auteur de nombreuses situations où l'ambassadeur est susceptible de rencontrer l'incertitude. La remise des instructions peut créer une béance entre l'ambassadeur et son mandant, le voyage également. À destination, l'exposé par l'émissaire de la proposition ne doit pas être dubitative. Le doute à l'étranger peut aussi s'immiscer en cas de discrimen entre les instructions et les circonstances, dans les entretiens, dans la communication des instructions, dans la conclusion, au départ. Le doute, l'incertitude, le risque, le péril sous diverses formes hantent véritablement l'action des ambassadeurs pour Bernard de Rosier.

1. Ont notamment été discutés les ouvrages suivants : Gesa Wilangowski, Frieden schreiben im Spätmittelalter. Vertragsdiplomatie zwischen Maximilian I., dem römisch-deutschen Reich und Frankreich, Berlin, 2017; Friederike Willasch, Verhandlungen, Gespräche, Briefe. Savoyisch-französische Fürstenheiraten in der Frühen Neuzeit, Ostfildern, 2018; Cornelia Neustadt, Kommunikation im Konflikt. König Erik VII. von Dänemark und die Städte im südlichen Ostseeraum 1423-1435, Berlin, Boston, 2018; Cornel Zwierlein (dir.), The dark side of knowledge. Histories of ignorance, 1400-1800, Leyde, Boston, 2016; Niels May, Zwischen Fürstlicher Repräsentation und Adliger Statuspolitik: das Kongresszeremoniell bei den westfälischen Friedensverhandlungen, Ostfildern, 2016. 
Afin de surmonter cette difficulté, il en appelle à des qualités traditionnelles, la patience, l'absence de témérité, le discernement, la prudence et l'expérience. L'ambassadeur, en possession d'un savoir pragmatique, non lettré, fondé sur l'expérience, la raison et son jugement, doit savoir écarter, diminuer l'incertitude, prévoir le futur, donner une impression de certitude et de confiance à ses interlocuteurs, savoir se comporter de manière adéquate dans les situations ambiguës. Rosier souligne la tension récurrente entre la nécessité de maintenir et d'entretenir la confiance d'une part et, de l'autre, l'exigence de faire avancer sa position sans révéler ses vues de façon trop nettes. Il ne s'agit donc pas seulement de combattre une incertitude jugée inefficace ou nuisible, mais de l'intégrer de façon graduée comme une part inhérente au travail diplomatique.

L'enjeu demeure en revanche très secondaire sous la plume d'Étienne Dolet. La possibilité de situations potentiellement problématiques pour des ambassadeurs exposés à des circonstances adverses est envisagée, mais seule la prudence des émissaires est proposée en guise de remède.

Quelques années plus tard, Konrad Braun consacre des développements plus étoffés à la question. Comme Rosier, il se méfie profondément des situations d'incertitude ou de doute, et cherche à les dominer. Dans une réflexion formellement assez différente, plus typologique et juridique, Braun reconnaît aussi, à sa manière, que tout n'est pas parfaitement prévisible dans le déroulement des échanges diplomatiques. L'examen de ses Cinq livres a permis de montrer qu'il distingue trois domaines dans lesquels surgissent le doute (dubium) et les situations incertaines (incerta) : la délibération préalable pour les ambassadeurs, l'exécution de la mission que les formulae ne prévoient pas intégralement et, de manière plus originale, les responsa media, des réponses médianes ou moyennes. Dans les différentes configurations envisagées, le principe d'adaptation aux circonstances doit selon lui prévaloir, en conformant l'action aux préceptes de la foi. Néanmoins, Braun n'hésite pas, dans la partie concernant les réponses, à instrumentaliser le doute, à en faire une arme potentielle de déstabilisation des interlocuteurs diplomatiques.

Le dernier texte examiné, El embajador de Juan Antonio de Vera, se présente sous la forme de conversations entre deux amis qui se retrouvent dans un même jardin à trois reprises. L'ambassadeur y est une figure au croisement de tensions contradictoires : il lui faut être chrétien, obéissant à son prince; viser la paix, mais parfois au prix de la guerre; obéir aux ordres, mais tenir compte des circonstances, des changements du temps. Sa tâche est selon Vera plus difficile que celle d'autres officiers, en raison de la distance, de la solitude, de la nécessité de saisir les intentions. Dès lors, pour un ambassadeur qui veut agir en conscience, les dilemmes, les occasions et les raisons de douter sont légion, et l'état d'ambassade s'apparente à une condition d'incertitude.

Pour y remédier, Vera formule des préceptes généraux : réduire au strict minimum toute possibilité de doute, d'incertitude ou d'ambiguïté entre le prince et l'ambassadeur; pour ce dernier, viser la certitude (atinar lo cierto), écarter l'incertitude à la manière d'un médecin, contruire donc des certitudes dans un environnement incertain; savoir avec le prince agir de façon à tirer profit de l'incertitude pour son maître. Ces préceptes lui paraissant néanmoins insuffisants, l'expérience et la pratique s'avèrent à 
ses yeux indispensables. D'où le choix d'une voie médiane visant à concilier des principes contraires, avec une forme d'obéissance tempérée, en usant de rhétorique et de comportements adaptés pour contourner les difficultés : fausse négligence, discrétion propice à susciter les révélations, prudence mêlée et acceptation, stoïque, du fait que le doute et l'incertitude sont inhérents au travail diplomatique.

Par des voies diverses - la réflexion juridique, la lecture humaniste des Anciens, l'histoire comme magister vitae, l'expérience diplomatique - ces textes se rejoignent donc pour pointer une tension récurrente entre, d'une part, une aspiration à la certitude dans l'action, à un respect absolu de son mandat par l'ambassadeur, et, de l'autre, une pratique où les situations de doute et d'incertitude sont inéluctables. Pour les plus hardis - les plus conséquents - il s'avère dès lors nécessaire de savoir maîtriser le doute, voire d'en user à bon escient pour être efficace.

Programme de l'année 2019-2020 : L'expérience de l'incertitude dans les échanges diplomatiques (suite).

Engagée l'an dernier, l'enquête sur l'expérience de l'incertitude dans les échanges diplomatiques a été poursuivie dans deux directions complémentaires. L'étude des écrits sur l'ambassadeur a d'abord été complétée par l'examen de trois témoins essentiels, le De officio legati du Vénitien Ermolao Barbaro, le De legato libri duo de son compatriote Ottaviano Maggi et L'ambassadeur de Jean Hotman de Villiers, ainsi que par le commentaire d'un texte de moindre ambition composé par l'humaniste Pierre Danès ${ }^{2}$.

Comme l'ont montré plusieurs travaux ${ }^{3}$, le De officio legati de Barbaro résulte de l'imbrication complexe d'expériences et de réflexions plus théoriques, il a valeur de justification, témoigne de l'importance nouvelle reconnue à l'ambassadeur résident et à la valeur éminente du service du prince ou de l'État. La lecture de ce tratarello (V. Branca) dans la perspective de l'incertitude a permis d'observer une « inquiétude » comparable à celle mise en évidence l'an dernier en séminaire par Noëlle-Laetitia Perret à travers l'étude de ses correspondances. Barbaro envisage en effet dans le De officio legati successivement plusieurs espaces d'incertitude potentielle concernant l'action et les propos de l'ambassadeur. L'auteur les circonscrit par une même méthode, consistant à susciter une interrogation intérieure susceptible de fournir à

2. Pierre Danès, Conseils à un ambassadeur, s.1., 1561, éd. L. Delavaud, Revue d'histoire diplomatique, 29 (juin 1915), p. 607-612.

3. Voir notamment Garrett Mattingly, Renaissance Diplomacy, Londres, 1955; Ermolao Barbaro, De Coelibatu. Officio legati, édition critique par Vittore Branca, Florence, L.S. Olschki, 1969; Vittore Branca, La sapienza civile: studi sull'Umanesimo a Venezia, Florence, L.S. Olschki, 1998; Bruno Figliuolo, Il diplomatico e il tratattista. Ermolao Barbaro ambasciatore della Serenissima e il "De officio legati”, Naples, Guida editori, 1999; Three Speeches by Venetian Ambassadors 1433-1486, éd., introd. et comm. Jan Rothkamm, Wiesbaden Harrassowitz, 2016; Dante Fedele, Naissance de la diplomatie, Baden-Baden, Nomos, 2017, ad indicem; et les travaux en cours de Noëlle-Laetitia Perret sur la correspondance d'Ermolao. 
l'ambassadeur une clef, un chemin le menant à la vérité de l'action au service de sa République. De la sorte, l'ambassade, charge au service de l'État, constitue également une épreuve d'incertitude pour l'homme vertueux qui la résout par la réflexion et par l'action, en appliquant des préceptes qui soutiennent la République et ses citoyens.

Ottaviano Maggi, dans le De legato duo, témoigne d'une grande sensibilité à l'imprévu et à ses dangers. Confiant dans la connaissance, dont il tisse sans cesse l'éloge, il recommande l'exercice de la dialectique et une approche pragmatique de l'occasion, combinant les arguments des Anciens, les valeurs chrétiennes et l'expérience, de façon à réduire l'incertitude, sans mettre en péril l'honnêteté de l'ambassadeur, ses vertus. Aussi l'incertitude demeure-t-elle à ses yeux fondamentalement une contrainte à abolir, à saisir par une forme d'omniscience pragmatique confinant à la divination; elle n'est pas, ou pas complètement, un outil dont il faut jouer dans les négociations, et jamais un moyen pour manipuler autrui.

L'analyse de L'ambassadeur de Jean Hotman de Villiers a permis de suivre une approche de la diplomatie caractérisée par un refus affiché de la figure de l'ambassadeur idéal, la recherche, paradoxale, de qualités et de vertus correspondant souvent de près au modèle ainsi décrié, et une attention extrêmement vive aux circonstances de l'action diplomatique, à la nécessité de l'adaptation. La place du doute, de l'ambiguïté et de l'incertitude dans les affaires diplomatiques est apparue importante dans ce texte. Hotman de Villiers reconnaît notamment, avec une forme de désenchantement accentuée à mesure des éditions (en particulier avec l'intégration de l'avis en 1613), la nécessité non plus seulement de maîtriser l'incertitude, mais aussi de jouer avec le certain et l'incertain.

Après un premier bilan général sur la place et le rôle dévolus à l'incertitude dans les écrits sur l'ambassadeur de la deuxième moitié du $\mathrm{Xv}^{\mathrm{e}}$ siècle au début $\mathrm{du}$ $\mathrm{XVII}^{\mathrm{e}}$ siècle, le séminaire a été consacré à l'étude de la diplomatie de la ville de Barcelone lors des retours de peste du $\mathrm{XV}^{\mathrm{e}}$ siècle. Dans cette optique ont été présentés et commentés des documents issus des registres de délibération des Archives historiques de la ville de Barcelone (série 1BII), des lletres closes expédiées par la capitale catalane (série 1BVI), des lettres reçues de princes et de rois, des lettres communes originales envoyées par les ambassadeurs et les représentants de la ville aux Corts (série 1BX), et des mesures prises par les juntas de peste (1CV11/1-5). Un épisode de peste très violent, qui frappe la ville d'août 1475 à octobre 1476, a plus particulièrement retenu l'attention. Il est apparu que l'épidémie ne provoque pas une fermeture complète de la cité, mais redéfinit les priorités et les critères des échanges : tandis qu'il devient pour les simples citoyens et a fortiori pour les forains plus difficile d'y pénétrer, les échanges politiques avec l'extérieur se poursuivent de façon intense avec la Péninsule, en évitant Valence et Majorque contaminées, pour se concentrer sur les relations avec le roi, les Corts, la Catalogne, au détriment des rapports avec les puissances étrangères, notamment avec les grandes cités marchandes de Méditerranée occidentale, les principales interlocutrices alors de la ville de Barcelone, suivant les routes des marchands et la carte des consulats. Les dernières séances ont mis en évidence la résilience de la diplomatie urbaine dans le contexte épidémique, le maintien d'une politique d'information très active, son adaptation dans une situation de grande incertitude, et la manière dont la peste même peut alors servir d'argument dans les 
échanges avec des puissances étrangères, voire se transformer en enjeu de luttes rhétoriques et diplomatiques. Pour la fama de Barcelone, ardemment défendue dans les correspondances avec l'étranger, il s'avère essentiel que la ville paraisse libérée de la peste, au prix, parfois, d'arrangements avec la réalité d'une situation épidémique qui n'est pas encore parfaitement sous contrôle. 